\title{
The Ageing Retina: Physiology or Pathology
}

\author{
JOHN MARSHALL
}

London

\begin{abstract}
Summary
The human retina is a unique component of the nervous system in that throughout life it is continuously exposed to optical radiation between 400 and $1400 \mathrm{~nm}$. The physiology of the ageing retina and the regression in visual performance with age cannot therefore be studied in isolation, or discriminated, from the life long cumulative effects of radiant exposure. This paper describes the spectrum of age related changes in the retina as they merge imperceptibly between declining visual function and overt pathology.
\end{abstract}

\section{Functional Loss}

Virtually every measure of visual function demonstrates declining performance with increasing age. ${ }^{1}$ However, since all the components of the eye age in different ways it is essential when assessing changes in retinal or neural function to allow for changes in the stimulous incident upon the retina due to either perturbations in the optic media or physiological processes in the anterior eye. For example, in the older eye the retinal irradiance achieved by a given source with a given geometry will be less than that in a young eye because of a reduced pupillary diameter (senile miosis), and decreasing transmission and increasing fluorescence properties of the cornea and lens. The latter will also modify the spectral irradiance as absorption and scatter in the ageing lens and cornea are more significant for shorter wavelengths. ${ }^{2}$

The practical difficulties in relating a particular functional loss to a specific anatomical site are slowly being resolved. For example McFarland and colleagues ${ }^{3}$ demonstrated a two times elevation of threshold in the dark adapted eye for every year over the age of 20 . Subsequently Gunkel and Gouras ${ }^{4}$ showed that this elevation resulted from two com- ponents, the larger one being diminished transmission by the ageing lens and a smaller one induced by neuronal changes in the ageing retina and cortex.

Age related degradations in visual acuity ${ }^{5,6}$ and contrast sensitivity ${ }^{7,8}$ have been documented for many years ${ }^{9}$ and are especially evident over the age of 60 years. ${ }^{5}$ Recently the use of sinusoidal grating patterns generated by a laser interferometer ${ }^{10}$ has enabled the independent assessment of degradation of the image quality by the optic media and the effective loss in resolving power of the neuronal components in the visual system. ${ }^{11,12}$ The conclusion from such studies is that the age related deterioration in contrast sensitivity is primarily caused by changes within the neuronal elements of the visual system. ${ }^{12}$

\section{Ageing Populations}

Given the increasing socioeconomic importance of rapid assimilation of visually communicated information any impairment of vision severely handicaps the performance of everyday tasks. As the handicap progresses economic and social dependence of the affected individual increases accordingly. Whilst a detailed analysis of population 
dynamics is beyond the scope of this article it is worth highlighting some worrying trends in the industrialised world. In many western countries an increase in life expectancy coupled with a falling birth rate is giving rise to ageing populations, for example, by the end of this decade 15.5 per cent of the population of the United Kingdom will be over the age of $65 .{ }^{13}$ Within this population there will be 2 million more women than men, a finding of significance as there is evidence that many senile eye conditions have an earlier onset and a higher incidence in women. ${ }^{14}$ Perhaps more worrying is the fact that the elderly constitute the major patient load for ophthalmic practitioners as there is a dramatic rise in the incidence of ocular pathologies in this group. In a recent study based on government sources the figures for blindness in England and Wales were quoted as less than 10 per 100,000 in the age group $0-4$ years and rising to 5,200 per 100,000 in the over 75 year olds. ${ }^{15}$ The figures whilst disturbing in themselves become much more worrying when extrapolated on the basis of the Melton Mowbray study ${ }^{16}$ which would suggest that blindness in the elderly is underestimated.in government figures by at least one third. Of this elderly blind patient group approximately 20 per cent will have been registered because of cataract, but between 46 and 50 per cent will be the result of macular disease. In essence senile macular degenerations are now the leading cause of blindness in the western world.

\section{Cellular Ageing}

The ageing process is usually monitored by reference to a single biologically independent variable, the passage of time. ${ }^{17}$ As a result it is often mistakenly considered as a solitary process. This is clearly not the case as at a cellular level even the most simplistic approach must recognise the differences between those cell systems undergoing cell division throughout life and those systems that do.not. For clarity we can designate the former cells as 'Dividing' and the later to 'Non-dividing' categories. Dividing systems are usually relatively simple cells and are found in locations where cells are under constant attack from physical agents, such as surface cells of skin or cornea, or cells lining the gut. The rate of cell replacement in such systems is phenomenal with entire cell populations changing every one to six days depending upon the tissue. Each day, some $500,000,000,000$ cells are shed and replaced throughout the body, and in an average lifetime cell populations may renew more than 12,000 times. In contrast cells in non-dividing systems are usually found in highly differentiated forms and do not undergo nuclear division after their initial development in early embryonic life.

A special problem occurs when a rapidly resynthesising cell system is trapped between two non-dividing systems. In such a situation one or both of the non-dividing systems may suffer secondary damage as a result of coping with the waste products generated by the trapped dividing system.

\section{Molecular Ageing}

At a molecular level both cell systems are undergoing constant renewal and replacement. Indeed it is this very process of continuous self synthesis that defines life (i.e. the maintenance of biological integrity). The only exception to this process of constant molecular formation and degradation is the genetic material itself, DNA, which in non-dividing systems is perfectly stable. However even here if a lesion occurs in the double helix then special enzymes detect it, remove the damaged part and attempt to replace it. This unique process of molecular repair rather than replacement has only been described for DNA, and unlike the constant process of molecular replacement it is only initiated by the presence of damage in the double helix. Continued cell life is dependent on not exceeding or overwhelming this repair process. DNA damage may result from a cell being irradiated by high energy photons in short wavelength light or ultra violet radiation and the repair process may slow down with age. Both of these concepts will be discussed later.

Molecular replacement is the cell's answer to the principle of entropy whereby all natural processes strive to dissipate energy and thereby exist in an increasing state of disorder. Any living cell is a highly ordered system and expends large amounts of energy 
to retain its ordered structure by constantly renewing it.

The rates of molecular renewal vary dramatically for example the aqueous humour is renewed every 1 to 1.5 hours. ${ }^{18}$ In contrast the proteins incorporated in rod disc membranes last for two weeks ${ }^{19}$ whilst those constituting the axoplasmic framework in a human retinal ganglion cell may exist for 3 months or more. In the latter case during their lifetime they slowly migrate from their site of synthesis in the cell body to their site of destruction at their synapses in the optic tectum. ${ }^{20}$ Several factors also influence the rates of molecular renewal, including nutrition, temperature, and radiant exposure. The latter exerting its effect both in the establishment of diurnal rythms in biological organisms, and by inducing photochemical changes in macromolecular systems. Diurnal rythms operate at both a cellular and molecular level, and there are many reports in the literature describing cyclic fluctuation in mitotic activity and protein synthesis according to the time of day or night. ${ }^{20}$ Photochemical processes always create problems for cells as the reaction products or intermediaries are often toxic, however in most natural exposures the cell's defence mechanisms or renewal systems can cope with the extra stress. In the case of unnatural or excessive exposures these defences may be overwhelmed and permanent damage may ensue.

\section{Embryology}

The initial step in the ageing of any system is its embryonic development and for the retina the process really begins in the first few weeks of life. The macula is apparent by about 11 to 13 weeks and can be clearly seen as a densely pigmented area on transilluminating globes at this age. The pigment epithelial cells within this region are taller but less wide than those in other locations. ${ }^{21}$ They retain this unique aspect ratio throughout life and it probably accounts for the density of their pigmentation i.e. by concentrating pigment granules/unit area relative to the axis of incident light. The photo-receptor cells that overlay these macular pigment epithelial cells are also highly differentiated as at 13 weeks this is the only area in which cone cells have already devel- oped inner segments. In all other locations the presumptive photoreceptor cells are still undergoing mitotic division. For some as yet unexplained reason further development of macular photoreceptor cells then seems to cease, and those in the rest of the retina rapidly overtake them during the remaining months in utero. At birth the macular photoreceptors are the most poorly developed, and do not reach full maturity until at least four to six years post-partum. ${ }^{22}$

\section{The Adult Macula}

As ageing of the macula is the most significant clinical problem, discussion will concentrate on this region. The term macula means patch, and to some extent this conveys the clinician's dilemma in defining the boundaries of this region. Ophthalmoscopically an individual macula is considered to be an area centred on the fovea throughout which there is an altered light reflex when compared with the surrounding fundus. The origins of this altered light reflex are complex with contributions from an altered retinal thickness, densely pigmented retinal pigment epithelium and the presence of a yellow pigment, the macula lutea. ${ }^{23}$ The extent of the ophthalmoscopic macula is highly variable between individuals, but commonly occupies a $3 \mathrm{~mm}$ diameter zone, $4 \mathrm{~mm}$ temporal and $0.8 \mathrm{~mm}$ inferior to the optic disc, being directly on the optic axis of the eye. The anatomical macula may or may not lie within the same boundaries as the ophthalmoscopic region, as the former is defined as that area of the retina where the number of layers of nuclei in the ganglion cell layer exceed two.

At the centre of the macula is the fovea, a central concavity or pit formed by the radial displacement of the inner retinal layers during development. ${ }^{24} \mathrm{At}$ the rim of the foveal pit the retina is extremely thick $(300 \mu \mathrm{m})$ due to both the number of displaced intermediary neurones and also the large number of ganglion cells that relate to the fovea The sloping walls of the pit referred to as the foveal clivus, rapidly thin the retina to form the floor of the pit, or the foveola where the retina is at its thinnest $(150 \mu \mathrm{m})$. Both the rim and floor of the fovea give rise to ophthalmoscopically visible reflexes in the young adult. The former 
causes a brilliant, horizontally elliptical surface reflex called the foveal reflex, whilst the latter gives rise to a minified or spot-like foveola reflex.

The foveola is the only rod free area of the retina and is also the centre of the foveal avascular zone. Retinal capillaries are present in the inner retinal layers from about half-way up the slopes of the clivus. It is generally considered that both the thinning of the retina and the absence of blood vessels contribute to the high acuity attained by this area.

The foveal cones are highly specialised in a number of ways. First, in the young, their inner and outer segments are of extremely small diameter $1.5 \mu \mathrm{m}$ ) thus facilitating a close packing density. Secondly because these cells have to enter into synaptic association with horizontal cells and bipolars that have been radially displaced out of the foveola they have developed a long axonal-like component called the inner connecting fibre. These fibres run from the inner aspect of the nuclear region to the cells synapses in the outer plexiform layer. Collectively these fibres form the fibre layer of Henle, and it is within these elements that most of the macular pigment is located. ${ }^{25,26,27}$ Because of these long inner connecting fibres, foveal cones are the largest photoreceptor cells in the retina, some being over $500 \mu \mathrm{m}$ long from tip of their outer segment to their synaptic surface. ${ }^{25}$ Inner connecting fibres are particularly well demonstrated in isolated receptor cells (Fig. $1)$.

The differences in retinal pigment epithelial cells in this region have already been mentioned, but it should also be stated that Bruch's membrane and the underlying choroid are thicker in the posterior pole than in other regions of the eye and that the choriocapillaries is more extensively developed ${ }^{28}$.

\section{The Ageing Macula}

No single quality of the posterior pole of the eye remains constant during life, although individual age-related changes are of differing importance and portent ${ }^{29}$ Commonly the collapse of the vitreous gel in the fourth or fifth decade of life is followed by a detachment of the posterior vitreous from its perimacular attachments to the inner limiting membrane of the retina. ${ }^{30}$ At about this time the brilliant shimmering foveal and foveola reflexes of youth are lost, giving evidence of an altered topography of the retinal surface. This may arise in part due to shallowing of the walls of the foveal pit resulting from an age related loss of retinal neurones. A recent study on human optic nerve demonstrated that a loss of almost 50 per cent of retinal ganglion cells can be expected over a 70 year life $\operatorname{span}^{31}$ and it should be remembered that 50 per cent of these cells serve the macula.

\section{Photoreceptor Cells}

The light-sensitive portions of the rods and cones consist of photopigments distributed within a series of hollow 'coin like' membranes. In rods the membranes or discs are discrete units isolated from each other and the boundary membrane of the cell, with about 1000 discs in each rod. In cones the disc membranes are all joined together, being continuous with the boundary membrane of the cell. The action of light upon these membranes leads to the production of a number of toxic products such as free radicals.

\section{Rod Cells}

Both rods and cones attempt to limit the damaging effects of ageing and light by constantly renewing their discs. ${ }^{19}$ Rods have a highly efficient process whereby 1-3 discs are manufactured each hour and thus between 30 and 100 news discs are made each day. With successive disc production, units are progressively displaced down the outer segment towards the pigment epithelium. Such is the capacity of the system that the entire light sensitive portion of the rod cell is renewed every two weeks. In order to prevent large fluctuations in rod length, old discs are removed from the tips of the outer segment by the phagocytic action of the retinal pigment epithelium. This process seems to be initiated by the onset of light, i.e. rod tips are 'eaten' on awakening in the morning. However, it may also be mediated by hormones, as a diurnal rhythm of disc shedding is exhibited even in prolonged periods of darkness. To date, evidence suggests that the underlying diurnal rhythms are initiated within the eye, and are 


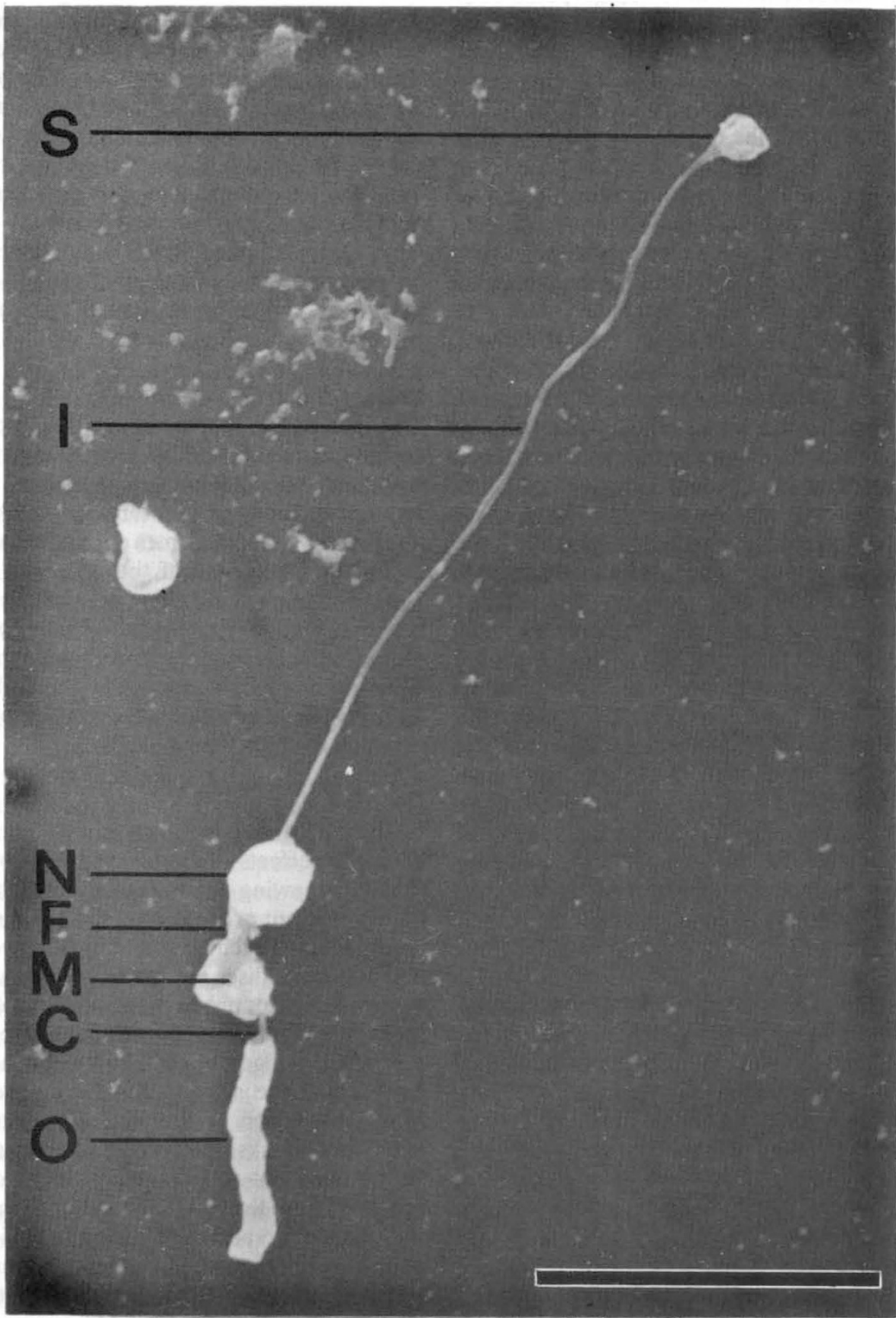

Fig. 1. Scanning Electron Micrograph of Isolated Rod Cell. The various portions of this photoreceptor can be easily identified as follows:

$s$ : synapse, $i$ : inner connecting fibre, $n$ : nucleus, $f$ : outer connecting fibre, $m$ : inner segment, $c$ : cilium, o: outer segment

The bar marker is $10 \mu \mathrm{m}$ 
not influenced by removal of the pineal gland. ${ }^{32}$

This rapid and continuous replacement of discs on an incremental basis, with each disc being a discrete unit in total isolation from its neighbours and the cell's boundary membrane, seems to confer adequate protection to the rod cells during the average human lifetime of 70 years. The result is that little evidence can be found of loss of rod cells in the ageing retina. Indeed so effective is the rod renewal system, that the only anomaly manifested with increasing age is a net increase in the numbers of disc membranes. ${ }^{33}$ This increase in discs is not accompanied by an increase in the axial length of the rod outer segment and in consequence, in most individuals over the age of 45 the rods develop convolutions in their outer segments. The increase in rod disc content infers that the rate of disc formation does not slow significantly with age; on the contrary, it would either suggest a slight acceleration in disc production or a decrease in the rate of phagocytosis; and as will be seen later the latter is probably the mechanism.

\section{Cone Cells}

Cones renew their discs at a much slower rate, with the best evidence suggesting that the whole system may take nine months to a year to renew. ${ }^{34}$ In contrast to rods, cone tips are 'eaten' at night. The apparently poor renewal rate of cone membranes may relate to the finding that cone 'discs' are not discrete, being joined together in a continuum. This conformation precludes individual disc replacement on an incremental basis. Molecular renewal is a random process therefore in such a fluid system as the cone outer segment membrane new molecules are just as likely to be replaced as old ones. Clearly this renewal mechanism is less efficient than that in rods and may account for the age-related changes observed in cones. In essence a small and approximately linear loss of cone membranes is seen between birth and 40 years and then a rate change occurs and membrane loss is more rapid. ${ }^{35}$ This loss of membrane order is eventually reflected in a net loss of cone cells over the age of 40 and especially from the fovea. ${ }^{36}$ As has been seen in the eyes of retinitis pig- mentosa sufferers, a drop out of photoreceptor elements from the matrix is followed by an increase in diameter of those remaining. Typical figures for the diameter of cone inner segments available in the literature show a range of values from 1.2 to $4.5 \mu \mathrm{m} .{ }^{37}$ We have shown that this distribution is age related, and that the rate of increase in cone diameter and presumable cell loss accelerates after the age of 40 years (Fig. 2).

Light absorption in photoreceptor cell outer segments is directly related to the concentration and orientation of molecules of visual pigments and this in turn is dependent upon the number and size of photoreceptor discs and their orientation. Both progressive lamellar disorientation and cone cell drop-out would contribute to diminished light absorption by visual pigments within the fovea with increasing age. Such a finding has recently been reported in subjects between the ages 22 and 50 years by a group using the technique of television ophthalmoscopy to measure cone pigment density difference spectra. ${ }^{38,39}$

It is also interesting to note that acquired defects of colour vision in particular in bluegreen discrimination are often associated with age or disease, the so-called Kollner's rule..$^{40}$ Such acquired defects are particularly noticeable in diabetics, and subsequent to peripheral retinal ablation by xenon or argon photocoagulators an acute increase in severity of the effect is noted ${ }^{41}$ and is thought to be related to the massive doses of scattered intraocular light. Whether the defects in blue perception result from a greater sensitivity of short wavelength cones to ageing, light or disease, or whether they become apparent in this type of photoreceptor because there are fewer of them in the cone population, is unclear.

\section{Retinal Pigment Epithelium}

Once inside the retinal pigment epithelium, the groups of engulfed discs called phagosomes undergo lysis. Some of the breakdown products are recycled but many are voided into the choroidal blood supply via Bruch's membrane. The pigment epithelium is a nondividing system and with increasing age there is a net loss of cells from this layer with a resulting increase in size of those remaining. 


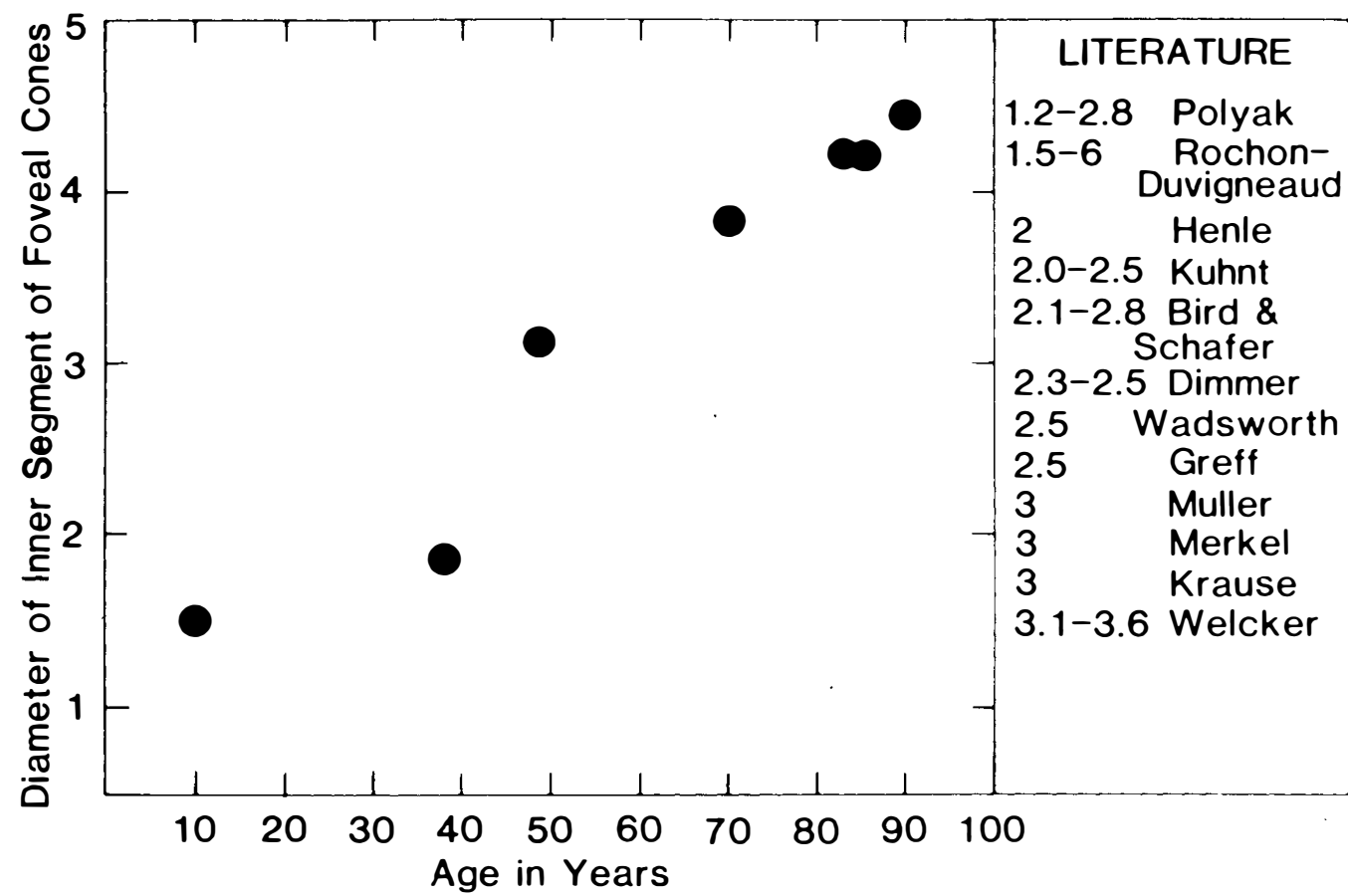

Fig. 2. Graph showing the increase in diameter of inner segments of foveal cones with increasing age. The literature data is from Polyack. ${ }^{37}$

Coupled with this reduction in available cells, both the lytic and voiding capabilities of those remaining seems to decline and result in the accumulation of incompletely degraded phagosomal particles called lipofuscin granules. ${ }^{42}$ In the absence of disease, lipofuscin becomes apparent within the pigment epithelium by the age of 10 years, and by the age of 40 years occupies some 8 per cent of the cytoplasmic volume of the cells. ${ }^{43}$ By 80 years this figure has risen to more than 20 per cent. As senescence represents the accumulation of abnormal or functionless molecules the build up of lipofuscin within the pigment epithelium is a clear example of an ageing cell.

Recently some interesting observations have been obtained from studies on human retinal pigment epithelial cells grown in tissue culture. First it is well established that the rate of cell division in culture depends upon the age of the donor from whom the cells were obtained, with fastest growth curves being displayed by cells from the young. ${ }^{44.45}$ The new observation is that in any given individual, cells derived from the macula grow less well than those from the retinal periphery, and that this differential increases with age. ${ }^{46} \mathrm{~A}$ major difference between cells obtained from these two locations is that those from the macula contain more lipofuscin than those from the periphery. ${ }^{47}$ In a further series of experiments Boulton and Marshall have shown that if pigment epithelial cells of any age are artificially fed lipofuscin granules in their culture media, cell division and metabolic activities are reduced as lipofuscin content increases, and that beyond a certain critical level the cells die. ${ }^{48}$ From these studies it would appear that the onset of senescence in the retinal pigment epithelium is at least in part due to the accumulation of lipofuscin which in turn reflects the demands made upon it by the constant phagocytic load of photoreceptor cell membranes.

Unlike any other macrophage which has a transient existence and having fulfilled its role by ingesting its target material dies to be replaced by a new cell, the same pigment epithelial cell must continuously engulf some three hundred million million discs during a 70 year life span. At about the age of 40 years the pigment epithelial cells become too full with 
these waste products and they attempt to rid themselves by pushing them out through Bruch's membrane.

\section{Bruch's membrane and choroid}

Bruch's membrane is an acellular membrane that is usually subdivided into five substrata, of which two are basement membranes, two are collagenous and the remaining one is composed of elastin. A useful analogy is to consider the fibrous layers as the wires in a garden sieve, however, in this case with increasing age, the fibrous components increase in numbers. Thus the sieve of Bruch's membrane assumes a progressively increased resistance to the passage of particles. This increased resistance, coupled with an increased outflow of cellular detritus, results in an accumulation of debris which in turn tends to displace the overlying pigment epithelial cells. Over the age of 40 years, focal aggregations of such sub-pigment epithelial debris are observed and these excrescences on Bruch's membrane are called Drusen. ${ }^{49}$

As well as the alterations induced in Bruch's membrane by phagocytic problems of the retinal pigment epithelium other ageing changes occur which are also thought to have functionaf significance, and of these the most prominent is the accumulation of a fine granular deposit on its innermost margin, termed by Sarks the 'Basal Linear Deposit' ${ }^{49}$ This deposit is a thickened and chemically modified basement membrane of the pigment epithelium. In association with these basement membrane changes the basal border of the epithelial cells undergo a reduction in the complexity of its convolutions, a finding that would suggest a progressive decline in active transport with age.

Within the fibrous layers of Bruch's membrane changes are also apparent. ${ }^{52}$ The collagen fibres become more numerous with age and there is an increasing incidence of fibres displaying an atypical banding periodicity. The fibre numbers also increase in the elastic layer, but here calcification is the most worrying feature as the resultant changes in elasticity renders Bruch's membrane more brittle. ${ }^{53}$ Such changes extend throughout the thickness of Bruch's membrane to involve the intercapillary pillars of the adjacent choriocapillaris which themselves increase in both axial and lateral dimensions and effectively reduce the size of intervening capillaries. As virtually all of the nutrition of central cones derives from the choroid, this combination of a greater volume of intervening material together with greater separation of elements of choriocapillaris undoubtedly contributes to a further decline in metabolic transfer capacity.

There are also some indications that perturbations in molecular transport may be selectively affected as biochemical studies have shown that the proteoglycans in Bruch's membrane change with age ${ }^{54}$ and in other basement membranes where such changes occur negatively charged fields are established and these impede the passage of similarly charged macromolecules. ${ }^{55} \mathrm{~A}$ further consideration in relation to retinal fluid control is that the net flow of water is from retina to choroid, with the pump residing in the pigment epithelium. If the build up of membranous debris within Bruch's membrane with age is predominantly lipid then a hydrophobic barrier may be created which would preferentially impede this outward movement of water from the retina. ${ }^{56,57}$

\section{The pathology of the Ageing Macula}

All of the previously described age related changes in the physiology of the macula may ultimately contribute to a pathological state. Some of the consequences of senescence are described below.

\section{Drusen}

All eyes over the age of 40 have the precursors of Drusen within Bruch's membranes in the peripheral retina. In such locations, Drusen do not seem to lead to sight-threatening sequelae. ${ }^{50}$ However, if they occur in the central retina they are identified as risk factors associated with more significant retinal pathology. ${ }^{51}$ If, as is presently postulated, Drusen result from the age related inability of the pigment epithelial cells to fully digest rod outer segment debris then the macular distribution of Drusen is easy to explain. If the geographic distribution of rods determined by Osterberg $^{52}$ is compared with the distribution of lipofuscin determined by Wing and Col- 
TEMPORAL

NASAL

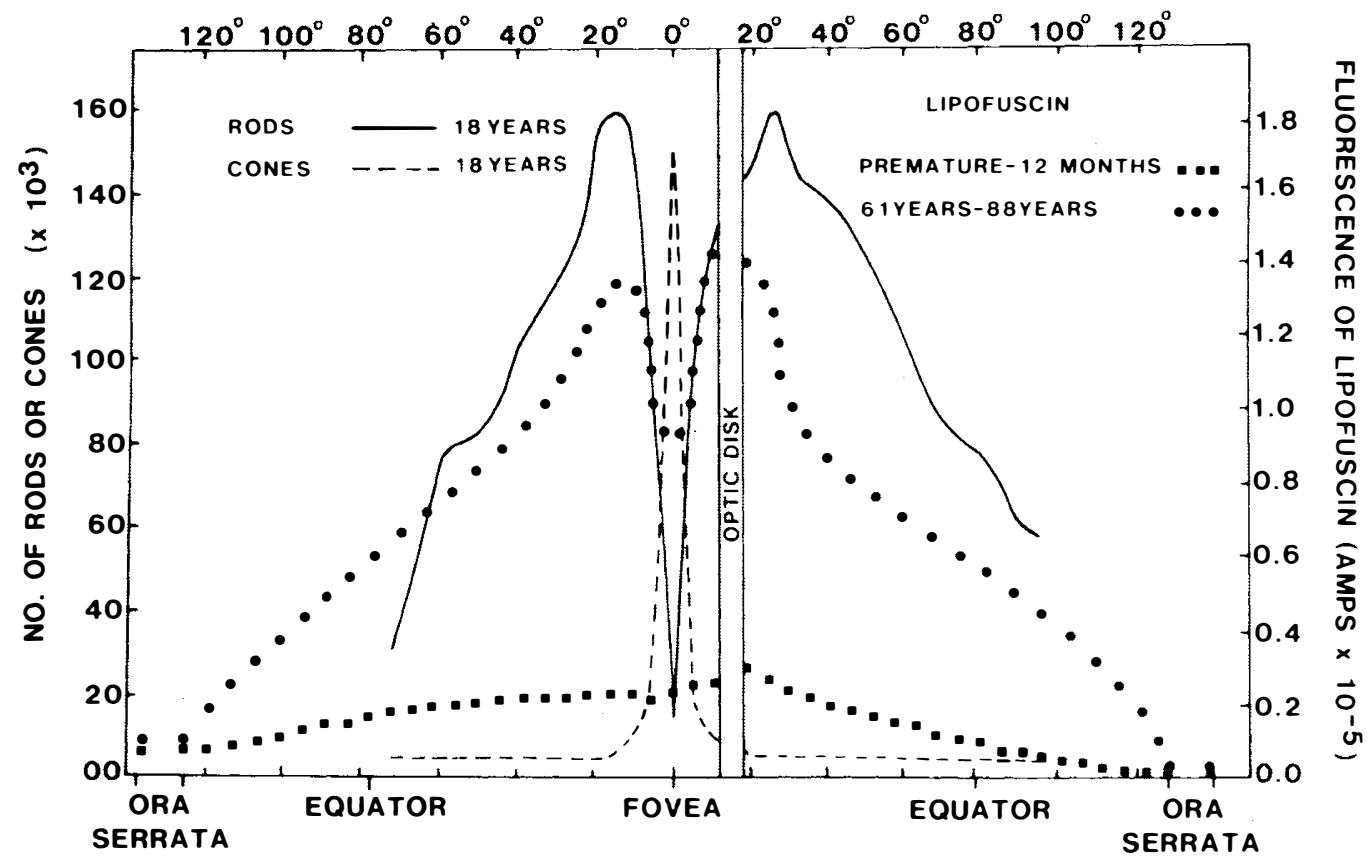

Fig. 3. Graph to show the relative topographic distributions of rods and cones in relation to the distribution of lipofuscin. The rod and cone distribution is taken from the workd of Osterberg ${ }^{58}$ whilst that of lipofuscin is taken from the work of Wing and colleagues. ${ }^{47}$ It should be noted that the maximal distribution of lipofuscin coincides with the maximal distribution of rods.

leagues $^{47}$ then the two can be seen to be coincident (Fig. 3).

\section{Retinal pigment epithelial detachment}

Here, in relation to, or exacerbated by, Drusen or sometimes as a result of other causes such as a thickened basal linear deposit, the pigment epithelium detaches from Bruch's membrane. If a hydrophobic barrier is present within Bruch's membrane ${ }^{57}$ and if the predominant flow of fluid is from retina to choroid then fluid will pool between the pigment epithelium and Bruch's membrane. With increasing time the pigment epithelium will literally pump itself off Bruch's membrane. ${ }^{56}$ If sufficient fluid accumulates under the area of detachment, or if the pigment epithelial barrier changes, or if the epithelium tears, the overlying photoreceptors may die as a result of the induced disturbances in homeostasis.

\section{Senile macular degeneration}

Senile macular degeneration (SMD) again results from the metabolic disadvantage resulting from the abnormal accumulation of debris between the pigment epithelium and the choroidal blood supply. In this case the macular and foveal photoreceptors atrophy. The factors controlling the underlying aetiology are not known but several authorities suggest a strong familial or genetic influence, ${ }^{51,59}$ Senile macular degeneration is the commonest form of macular disease and is frequently bilateral. ${ }^{16}$ Whilst epidemiological studies are poor, a recent survey in the USA gave a prevalence for both eyes of 4 per cent between 65-74 years and 17 per cent between $75-85$ years, with a 22 per cent prevalence for single eyes in the older group. ${ }^{60}$ In the United Kingdom, in the over-70 year age group, senile macular degeneration is responsible for as many as 47 per cent of blind registrations ${ }^{61}$ and 63 per cent of low vision cases. ${ }^{62}$ In all studies, except those in Australia ${ }^{63}$ women 
show a higher prevalence than men with an earlier onset. Given the ageing populations of the western world and the longevity of women, there will be a marked increase in the numbers of people suffering from senile macular degeneration. Unless treatment or prophylatic measures improve, we must prepare for the social and economic consequences of an increasing blind population.

\section{Disciform lesions}

There is a further complication in senile macular degeneration in which first macrophages and then choroidal blood vessels begin to invade Bruch's membrane. These vessels may penetrate the pigment epithelium and thereby circumvent its blood retinal barrier properties and they may also rupture and bleed with diastrous consequences for vision. ${ }^{64.65}$ They also have an unfortunate behavioural property in that whatever their site of origin they tend to grow towards the fovea where their leaky properties cause rapid atrophy of the photoreceptor cells. Currently laser treatment is only really effective if the patients are diagnosed very early in the evolution of the lesion and recurrences are such that one estimate is that only between $300-400$ people are kept off the blind registration per year for all the treatment given for SMD.

\section{The role of light in relation to retinal ageing}

The human retina is normally subjected to irradiances below $10^{-4} \mathrm{Wcm}^{-2}$ and above this level the exposure duration is limited by blinking. We do not know if the changes in our light environment over the past 50 years are affecting retinal performance or disease processes; we do know that artificial light regimes have dramatic effects on the retinae of experimental animals ${ }^{66}$ and that in commerce and industry, recommended levels of illumination are constantly being revised upwards. ${ }^{67.68} \mathrm{We}$ also know that in the clinical environment we have a worst-case situation with extremely bright lights and no functional physiological protective mechanisms, i.e. the blink reflex and aversion responses are commonly overcome by anaesthesia and pupillary closure by mydriasis.

The changes in the retina induced by excessive exposure to light are complex to under- stand and it is difficult even to relate the site of primary damage to a particular cell type, as several apparently conflicting pieces of empirical evidence have to be considered. First it should be explained that, as with any biological reaction, a spectrum of lightinduced changes can be expected and that particular changes within such a spectrum will be favoured by particular exposure regimes. Secondly, because of lack of knowledge of the total spectrum, present accounts may tend to polarise and isolate damage mechanisms in a way which may subsequently prove unjustified. At present, experimental data exist for four different types of exposure regimes and these seem to indicate the existence of two different mechanisms. The exposure regimes are in order of increasing irradiance:

(a) prolonged exposure to commercial or domestic levels of fluroescent lighting (several hours to several days). ${ }^{69,70}$

(b) repetitive exposure to narrow band monochromatic lights (several hours/ day for some days)..$^{71}$

(c) low level non-thermal exposure to lasers $(10 \text { seconds to several minutes })^{72}$ and

(d) relatively short exposures to clinical light sources (several minutes). ${ }^{73}$

Regimes (a) and (b) result in damage that could be explained by absorption of light by visual pigments in the photoreceptor cells; whilst (c) and (d) seem to be dependent upon absorption within the pigment epithelium.

Photoreceptor cells. The initial indication that moderate levels of illumination from artificial light sources could result in retinal damage came in the mid-sixties when it was shown that if rats were kept under constant illumination from fluorescent lamps, their photoreceptor cells degenerated. ${ }^{74}$ The original work of Noell and others was criticised because of the use of rats, a nocturnal animal with a predominantly rod retina. However, when such studies were extended to diurnal species a further surprising finding was made in that cones were affected at irradiances lower than those required to damage rods. ${ }^{70}$ For example in the monkey, $12 \mathrm{~h}$ exposures with retinal irradiances between 195 and 361 $\mathrm{Wcm}^{-2}$ damaged cones, whilst irradiances of $361-615 \mathrm{Wcm}^{-2}$ were required to produce rod 
damage.$^{70}$ These studies also showed that the fovea was apparently the most sensitive area; however, it should be remembered that in primates this region of retina is said to recline the highest irradiance. This rod-cone differential is almost certainly not a difference in threshold to primary damage, but probably results from the different repair capacity of the two cell types, i.e. rods and cones are probably damaged to the same extent but rods are better able to repair themselves and survive. Two further points should be emphasised; first all these experiments were carried out on young healthy animals and effects on the cones of age-compromised retinae may be far more dramatic. Secondly, light damage from fluorescent lamps always becomes more severe when exposure periods are sufficiently long to eliminate the normal diurnal cycle of light and dark. ${ }^{75}$ This may have interesting implications for succeeding generations as fluorescent lights now gives us unlimited access to daylight levels of illumination.

In a second type of experiment, exposing monkeys to repetitive flashes of light from monochromatic sources, Harwerth and Sperling ${ }^{71}$ showed that cone loss was most easily elicited by exposure to blue light; a finding which perhaps explains Kollner's rule? Thus far then, light-induced damage has been described in photoreceptor cells, occurring in cones at lower irradiances than rods, and in short wavelength exposure at lower irradiances or shorter exposure durations than for long wavelengths.

Pigment epithelium. The second group of results that are attributed to photochemical mechanisms relate to relatively higher retinal irradiances delivered over shorter time spans. These studies show that for supra-threshold exposures only, in the $10 \mathrm{~s}-2 \mathrm{~h}$ exposure domain, the initial changes occur in the pigment epithelium. Detailed studies with lasers demonstrated a clear relationship between thresholds for such damage and wavelength, with again lower irradiances being required to produce damage with short wavelength radiation. ${ }^{72,76}$

Ham's data has resulted in most national and international bodies concerned with laser safety incorporating wavelength-related restrictions on prolonged viewing of low-level laser light, the so called 'Blue Light Hazard'. ${ }^{77}$ It has also enabled the relative retinal hazard of any light source to be more accurately assessed, as we now know that in addition to the total retinal irradiance achieved by a source, the relative spectral emission also has to be considered in relation to Blue Light Hazard weighting functions. ${ }^{78}$ In this context it is of interest that the absorption spectra of the ageing lens and that of the macular pigment seem beautifully designed to filter out the Blue Light Hazard before incident radiation reaches the light-sensitive portions of the photoreceptor cells. ${ }^{79}$ Cataract surgeons should think very carefully of the possible problems of suddenly exposing an age-compromised retina to an influx of potentially hazardous wavelengths by removing an aged lens full of blue light absorbing yellow pigment, and should perhaps redress the consequences of their actions by the use of a suitably tinted artificial lens. ${ }^{80}$ The importance of the absorption properties of the lens in particular in relation to attenuating UVA, have been well demonstrated by Ham and his colleagues ${ }^{81}$ who demonstrated that in aphakic monkeys "blue light" like retinal lesions could be induced by UVA at energy levels six times lower between 325 and $350 \mathrm{~nm}$ than at the blue light peak of $441 \mathrm{~nm}$.

The current debate concerning whether or not intraocular lenses (IOLs) should or should not contain UVA blocking filters is confusing to the casual observer and much of the evidence quoted by the respective protagonists is ambiguous. The concern of the antifiltration group is 'do we have a treatment for a condition that doesn't exist, and for which the patient pays a commercial premium'. This group quotes the conflicting data on the incidence of cystoid macular oedema (CME) after cataract surgery in groups of patients with and without UVA blockers. CME is however not the critical pathology in relation to monitoring the performance of IOLs. CME is a relatively acute condition with multifactorial origins most of which relate to surgery. These can be summarised as factors due to vitreous manipulation, secondary inflammation, fluid mobility from anterior to posterior globe, and light exposure during surgery. If light is an important cofactor then the exposure to the 
microscope lamp during surgery is critical as this may initiate a subthreshold blue light or Ham type lesion to the retinal pigment epithelium. A compromised pigment epithelium with disturbed pump and barrier properties could well ultimately result in accumulation of fluid in the retina and CME. To prevent CME filtration of the microscope lamp is probably more important than a UVA absorber in the IOL. In contrast, SMD is a chronic condition rarely seen in eyes in which cataracts are present. In this case subsequent to surgery the retina is going to be exposed to high levels of blue light and in the absence of a filter, UVA. This sudden increase in short wavelength radiation may well accelerate photoreceptor cell damage due to photochemical processes of the non-Ham type. For example it has recently been reported from the Wilmer Institute (ARVO 1986) that in a group of monkeys in which non UV absorbing IOLs had been inserted in one eye, there was a three times higher incidence of macular drusen in the operated eyes. In this case a UVA filter in the IOL may well have delayed the onset of senile changes in the macula or at the very least offered an element of protection. The use of the UVA blocking IOLs is relatively new, but over the next few years studies on these patients should include the pattern and incidence of post operative SMD rather than $\mathrm{CME}$ as a guide to their usefulness.

\section{References}

${ }^{1}$ Kline D: Aging and the spatiotemporal discrimination performance of the visual system. Eye. 1987; 1: 323 .

2 Weale RA: Senescent vision: Is it all the fault of the lens. Eye. 1987; 1: 217.

${ }^{3}$ McFarland RA, Domey RC, Warren AB, Ward DC: Dark adaptation as a function of age. I. A statistical analysis. J. Gerontol. 1960; 15: 149-54.

${ }^{4}$ Gunkel RD, Gouras P: Changes in scotopic visibility thresholds with age. Arch. Opthalmol. 1963; 69: 4-9.

${ }^{5}$ Slataper FJ: Age norms of refraction and vision. Arch. Ophthalmol. 1950; 43: 466-81.

${ }^{6}$ Weale RA: Senile changes in visual acuity. Trans. Ophthalmol. Soc. U.K. 1975; 95: 36-8.

${ }^{7}$ Arden GB, Jacobson $\mathrm{JJ}$ : A simple grating test for contrast sensitivity: preliminary results indicate values in screening for glaucoma. Invest. Ophthalmol. and Vis. Sci. 1978; 17: 23-32.

${ }^{8}$ Skalka HW: Effect of age on Arden grating acuity. Br. J. Ophthalmol. 1980; 64: 21-3.

${ }^{9}$ Donders FC: On the Anomalies of Accommodation and Refraction of the Eye. 1864; London: New Sydenham Soc.

${ }^{10}$ Campbell FW, Green DG: Optical and retinal factors affecting visual resolution. J. Physiol. 1965; 181: 576-93.

${ }^{11}$ McGrath D, Morrison JD: The effects of ageing on the optical quality of the human eye. J. Physiol. (Lond.) 1983; 343: 81-2.

12 Morrison JD, McGrath C: Assessment of the optical contribution to the age-related deterioration in vision. Quart. J. Exper. Physiol. 1985; 70: 24969.

${ }^{13}$ OPCS. Office of Population Censuses and Surveys London, St. Catherine's House, 10 Kingsway, London, WC2B 6JP, U.K. 1983.

${ }^{14}$ Knave B, Tengroth B, Voss M: Age and sex distribution of some retinal macular diseases: senile and presenile macular degeneration and central serous retinitis. Acta Ophthalmol. (In Press).

${ }^{15}$ Royal National Institute for the Blind: Initial Demographic Study. Shankland Cox, London, 1985.

${ }^{16}$ Gibson JM, Rosenthal AR, Lavery J: A study of the prevalence of eye disease in the elderly in an English Community. Trans. Ophthalmol. Soc. U.K. 1985; 104: 196-203.

${ }^{17}$ Mainster M: Light and macular degeneration: A biophysical and clinical perspective. Eye. 1987; 1: 304.

${ }^{18}$ Macri FJ, Dixon RL, Rall DP: Aqueous humor turnover rates in the cat. Invest. Ophthalmol. 1965; 4: 927-34.

${ }^{19}$ Young RW: Visual cells and the concept of renewal. Invest. Ophthalmol. Vis. Sci. 1976; 15: 700-25.

${ }^{20}$ Young RW: The Bowman Lecture. Biological Renewal. Applications to the eye. Trans. Ophthalmol. Soc. U.K. 1982; 102: 42-75.

${ }^{21}$ Streeten BW: Development of the human retinal pigment epithelium and the posterior segment. Arch. Ophthalmol 1969; 81: 383-94.

22 Abramov I, Gordon J, Hendrickson A, Hainline L, Dobson V, LaBossiere E: The retina of the newborn human infant. Science 1982; 217: 265-7.

${ }^{23}$ Nussbaum JJ, Pruett RC, Delori FC: Historic perspectives. Macular yellow pigment. The first 200 years. Retina 1981; 1: 296-310.

${ }^{24}$ Hendrickson AE, Yuodelis C: The morphological development of the human fovea. Ophthalmology. 1984; 91: 603-12.

${ }^{25}$ Marshall J, Hamilton AM, Bird AC: Histopathology of ruby and argon laser lesion in monkey and Human Retina: A comparative study. Br. J. Ophthalmol. 1975; 59: 610-30.

${ }^{26}$ Snodderly DM, Brown PK, Delori FC, Auran JD: The Macular Pigment. I. Absorbance Spectra, Localisation, and Discrimination from other yellow pigments in primate retinas. Invest. Ophthalmol. Vis. Sci. 1984; 25: 660-73.

${ }^{27}$ Snodderly DM, Auran JD, Delori FC: The Macular Pigment. II. Spatial Distribution in Primate Retinas. Invest. Ophthalmol. Vis. Sci. 1984; 25: 67485.

${ }^{28}$ Gass JOM: Pathogenesis of disciform detachment of the neuroepithelium. 3. Senile disciform macular 
degeneration. Am. J. Ophthalmol. 1967; 63: 61744.

${ }^{29}$ Marmor MF: Aging and the Retina. In: Aging and Human Visual Function. R. Sekuler, D. Kline, J. Dismuths (eds.) New York: Alan R. Liss. Inc. 1982; 59-78.

${ }^{30}$ Sebag J, Balazs EA: Pathogenesis of cystoid macular edema: An anatomic consideration of vitreoretinal adhesions. Survy Ophthalmol. 1984; 28: 493-8.

${ }^{31}$ Balazs AG, Rootman J, Drance SM, Schulzer M, Douglas GR: The effect of age on the nerve fiber population of the human optic nerve. Am. J. Ophthalmol. 1984; 97: 760-6.

${ }^{32}$ Flannery JG, Fisher SK: Circadian disc shedding in Xenopus retina in vitro. Invest. Ophthal. Vis. Sci. 1984; 25: 229-32.

${ }^{33}$ Marshall J, Grindle CFJ, Ansell PL, Borwein B: Convolutions in human rods: An ageing process. Br. J. Ophthalmol. 1979; 62: 181-7.

${ }^{34}$ Foulds W: Factors influencing visual recovery in retinal detachment surgery. Trans. Ophthalmol. Soc. U.K. 1980; 100: 72-7.

${ }^{35}$ Weale RA: The eye and ageing. Interdisipl. Topics Geront. 1978; 13: 1-13.

${ }^{36}$ Gartner S, Henkind P: Ageing and degeneration of the human macula. 1. Outer nuclear layer and photoreceptors. Br. J. Ophthalmol. 1981; 65: 23-8.

${ }^{37}$ Polyak SL: The Retina. University of Chicago Press. 1941.

${ }^{38}$ Kilbride PE, Read JS, Fishman GA, Fishman M: Determination of human cone pigment density difference spectra in spatially resolved regions of the fovea. Vision Res. 1983; 23: 1341-50.

${ }^{39}$ Kilbride PE, Hutman LP, Read JS, Fishman M: The ageing human eye and cone pigment density difference in the fovea. Invest. Ophthalmol. Vis. Sci. 1984; 24: 198.

${ }^{40}$ Kolner M: Die Storungen des Furbensinnes ihre Klinische Bedeutung und ihre Diagnose, Karger, Berlin 1912.

${ }^{41}$ Birch J, Hamilton AM: Xenon arc and argon laser photocoagulation in the treatment of diabetic disc neovascularization. 2. Effect on Colour Vision. Trans. Ophthalmol. Soc. U.K. 1981; 101: 93-9.

${ }^{42}$ Feeney L: The phagolysosomal system of the pigment epithelium. A key to retinal disease. Invest. Ophthalmol. Vis. Sci. 1973; 12: 635-8.

${ }^{43}$ Feeney-Burns L, Hilderbrand ES, Eldridge S: Aging Human RPE: Morphometric Analysis of Macular, Equatorial and Peripheral Cells. Invest. Ophthalmol. Vis. Sci. 1984; 25: 195-200.

${ }^{44}$ Boulton ME, Marshall J, Mellerio J: Human retinal pigment epithelial cells in tissue culture: A means of studying inherited retinal diseases. 1982; 1816 March of Dimes Foundation.

${ }^{45}$ Flood MT, Gouras P, Kjeldbye H: Growth characteristics and ultrastructure of human retinal pigment epithelium in vitro. Invest. Ophthalmol. Vis. Sci. 1980; 19: 1309-20.

${ }^{46}$ Flood MT, Haley JE, Gouras P: Cellular Aging of
Human Retinal Epithelium in vivo and in vitro. Monogr, devl. Biol. 1984; 17: 80-93.

${ }^{47}$ Wing GL, Blanchard GC, Weiter JL: The topography and age relationship of lipofuscin concentration in the retinal pigment epithelium. Invest. Ophthal. Vis. Sci. 1978; 17: 601-7.

${ }^{48}$ Boulton ME, Marshall J: The effects of increasing numbers of phagocytic inclusions on human retinal pigment epithelial cells in culture: A model for ageing. Br. J. Ophthalmol. 1986; 70: 808-15.

${ }^{49}$ Sarks SH: Ageing and degeneration in the macular region: A clinico-pathological study. $\mathrm{Br} . J$. Ophthalmol. 1976; 60: 324-41.

${ }^{50}$ Foos RY, Trese MT: Chorioretinal junction: Vascularization of Bruch's Membrane in peripheral fundus. Arch. Ophthalmol. 1982; 100: 1492-503.

${ }^{51}$ Gass JDM: Drusen disciform macular detachment and dégeneration. Arch. Ophthalmol. 1973; 90: 208-17.

52 Grindle CFJ, Marshall J: Ageing changes in Bruch's membrane and their functional implications. Trans. Ophthalmol. Soc. U.K. 1978; 98: 172-5.

${ }^{53}$ Loffler KU, Lee WR: Basal linear deposit in the human macula. Graefe's Arch. Clin. Exp. Ophthalmol; 1986, 224: 502-6.

${ }^{54}$ Hewitt T, Newsome D: Altered synthesis of Bruch's membrane proteoglycans associated with dominant retinis pigmentosa. Current Eye Res., 1985; 4: $169-74$

${ }^{55}$ Kanwar YS, Farquhar MG: Anionic sites in the glomerular basement membrane: In vivo and in vitro localization to the laminae rarae by catronic probes. J. Cell Biol. 1979; 81: 137-53.

${ }^{56}$ Bird AC, Marshall J: Retinal pigment epithelial detachments in the elderly. Trans. Ophthalmol. Soc. U.K. 1986; 105: 674-82.

${ }^{57}$ Harper CA, Marshall J: The deposition of Lipids in Bruch's membrane and the implications for age related retinal pathologies. Br. J. Ophthalmol. 1987 (in press)

58 Osterberg GA: Topography of the layer of rods and cones in the human retina. Acta. Ophthalmol. 1935; 13: Suppl. 6; 1-102.

${ }^{59}$ Hyman LG, Lilienfeld AM, Ferris FL, Fine S: Senile macular degeneration: A case control study. Am. J. Eipdemiol. 1983; 118: 213-27.

${ }^{60}$ Khan MA, Leibowitz HM, Ganley JP, Kini MM, Colton T, Nickerson RB, Dawber TR: The Framingham eye study. I. Outline and major prevalence findings. Am. J. Expidem. 1977; 106: 17-32.

${ }^{61}$ HMSO. Blindness and partial sight in England 1969 1976. Reports on Public Health and Medical subjects No. 129. HMSO London 1979.

62 Robbins HG: Low vision care for the over 80's. Aust. J. Optom. 1981; 64: 243-51.

${ }^{63}$ Robbins HG: The low vision patient of tomorrow. Aust. J. Optom. 1978; 61: 54-64.

${ }^{64}$ Lee WR: Degenerative and other disorders of the retina and choroid. In Garner, $A$. and Klintworth, G. K. (Eds.) Pathology of Ocular Disease: A Dynamic Approach 1982; 1321-1351. Marcel Dekker, New York. 
${ }^{65}$ Eagle RC: Mechanisms of Maculopathy. Ophthalmology 1984; 91: 613-26.

${ }^{66}$ Lanum J: The damaging effects of light on the retina. Empirical findings, theoretical and practical implications. Surv. Ophthalmol. 1978; 22: 22149.

${ }^{67}$ IES Code: Recommendations for good interior lighting. Illuminating Engineering Society, London 1961.

${ }^{68}$ IES Code: Recommendations for lighting buildings. Illuminating Engineering Society, London 1968.

${ }^{69}$ Marshall J, Mellerio J, Palmer DA: Damage to pigeon retinae by moderate illumination from fluorescent lamps. Exp. Eye Res. 1972; 14: 164-9.

${ }^{70}$ Sykes SM, Robinson WG, Waxler M, Kuwabara T: Damage to the monkey retina by broad-spectrum fluorescent light. Invest. Ophthalmol. Vis. Sci. 1981; 20: 425-34.

${ }^{71}$ Hawerth RS, Sperling HG: Prolonged colour blindness induced by intense spectral lights in Rhesus monkeys. Science N.Y. 1971; 174: 520-3.

${ }^{72}$ Ham WT, Mueller HA, Sliney DH: Retinal sensitivity to short wavelength light. Nature 1976; 260: 153-5.

${ }^{73}$ Marshall J: Light damage and the practice of ophthalmology. In Rosen, ES, Maining, WM and
Arnott, EJ (Eds.) 1983; Chap. 19: 182-207. C. V. Moseby, St. Louis.

${ }^{74}$ Noell WK, Walker VS, Kang BS, Berman S: Retinal damage by light in rats. Invest. Ophthalmol. Vis. Sci. 1966; 5: 450-73.

${ }^{75}$ Hollyfield JG, Basinger SF: RNA Metabolism in the retina in relation to cyclic lighting. Vision Res. 1980; 20: 1151-5.

${ }^{76}$ Lawill T, Crockett S, Currier G: Retinal damage secondary to chronic light exposure. Documenta Ophthalmol. 1977; 44: 379-402.

${ }^{77}$ British Standards Institute: Radiation safety of laser products and equipment manufacturing requirements, user's guide and classification, BS.4803 1983.

${ }^{78}$ Sliney D, Wolbarsht M: Safety with lasers and other optical sources. Plenum Press, New York. 1980.

${ }^{79}$ Marshall J: Radiation and the Ageing Eye. Ophthal. Physiol. Opt. 1984; 4: 1-23.

${ }^{80}$ Mainster MA: Spectral transmittance of intraocular lenses and retinal damage from intense light sources. Am. J. Ophthalmol. 1978; 85: 167-70.

${ }^{81}$ Ham WT, Mueller HA, Ruffob JJ: Action spectrum for retinal injury from near-ultraviolet radiation in the aphakic monkey. Am. J. Ophthalmol. 1982; 93: 299-306. 